\title{
Probing the extragalactic cosmic rays origin with gamma-ray and neutrino backgrounds
}

\author{
Noemie Globus* \\ Racah Institute of Physics, The Hebrew University, 91904 Jerusalem, Israel \\ E-mail: noemie.globus@mail.huji.ac.il
}

\section{Denis Allard}

Laboratoire Astroparticule et Cosmologie, Université Paris Diderot/CNRS, 10 rue A. Domon et

L. Duquet, F-75205 Paris Cedex 13, France

E-mail: allardeapc.in2p3.fr

\section{Etienne Parizot}

Laboratoire Astroparticule et Cosmologie, Université Paris Diderot/CNRS, 10 rue A. Domon et L. Duquet, F-75205 Paris Cedex 13, France

E-mail: parizoteapc.in2p3.fr

\section{Tsvi Piran}

Racah Institute of Physics, The Hebrew University, 91904 Jerusalem, Israel

E-mail: tsvi.piran@mail.huji.ac.il

GeV-TeV gamma-ray and PeV-EeV neutrino backgrounds provide a unique window on the nature of the ultra-high-energy cosmic-rays (UHECRs). We discuss the implications of the recent FermiLAT data regarding the extragalactic gamma-ray background (EGB) and related estimates of the contribution of point sources as well as IceCube neutrino data on the origin of the UHECRs. We calculate the diffuse flux of cosmogenic $\gamma$-rays and neutrinos produced during the UHECRs propagation and derive constraints on the possible cosmological evolution of UHECR sources. In particular, we show that the mixed-composition scenario which is in agreement with both (i) Auger measurements of the energy spectrum and composition up to the highest energies and (ii) the ankle-like feature in the light component detected by KASCADE-Grande, is compatible with both the Fermi-LAT measurements and with current IceCube limits.

35th International Cosmic Ray Conference - ICRC2017

10-20 July, 2017

Bexco, Busan, Korea

${ }^{*}$ Speaker. 


\section{Introduction}

The interaction of ultra-high-energy cosmic-rays (UHECRs) with the photon backgrounds during their propagation in intergalactic space produces cosmogenic $\gamma$-ray photons and neutrinos $(v \mathrm{~s})$. The flux of these secondary messengers is highly sensitive to the spectral shape, maximal energy, composition and cosmological evolution of the UHECR sources, and therefore, one can derive important constraints on the UHECR origin from a multi-messenger approach that takes these into account (Protheroe \& Johnson, 1996; Coppi \& Aharonian, 1997; Ahlers \& Salvado, 2011; Decerprit \& Allard, 2011; Berezinsky et al., 2016; Supanitsky, 2016; Gavish \& Eichler, 2016, for $\gamma$-rays); (e.g. Stecker, 1979; Engel et al., 2001; Seckel \& Stanev, 2005; Allard et al., 2006; Anchordoqui et al., 2007; Ahlers et al., 2009; Kotera et al., 2010, for $v$ s).

The recent Fermi-LAT data (Ackermann et al., 2015), together with statistics of the photon counts in the skymap pixels (e.g. Malyshev \& Hogg, 2011, and references therein) have enabled different authors (Ackermann et al., 2016; Zechlin et al., 2016, hereafter A16 and Z16) to estimate the flux contributed by point sources (PS) well below the Fermi-LAT detection limits. These studies show that resolved and unresolved PS account for the majority of the EGB. Since a $\gamma$-ray background due to extragalactic cosmic rays (EGCRs) is unavoidable, it is crucial to verify that the proposed UHECR source models do not violate the existing constraints.

Recent measurements by the Pierre Auger Observatory (Auger) indicate that the composition of UHECRs is mixed (predominantly light) at the ankle of the cosmic-ray spectrum, and it gets progressively heavier as the energy increases (Aab et al., 2014). This composition trend can be interpreted as the signature of a low maximal energy-per-unit-charge $\left(E_{\max } / Z \lesssim 10^{19} \mathrm{eV}\right)$ of the nuclei accelerated at the dominant sources of UHECRs. Below $10^{18} \mathrm{eV}$, the KASCADE-Grande experiment reported an ankle-like feature in the energy spectrum of light (proton-helium) elements with a break at $\sim 10^{17} \mathrm{eV}$ (Apel et al., 2013; Bertaina et al., 2015). This "light ankle" can be naturally understood as the emergence of a light EGCR component, taking over the steeper Galactic cosmic-ray (GCR) component.

We discuss here the viability of a class of mixed-composition models in which the KASCADEGrande and Auger data are understood in terms of a transition between a GCR component and a single EGCR component with a soft proton spectrum and low $E_{\max }$. This soft proton component, responsible for the KASCADE-Grande light ankle, would be the dominant contributor to the cosmogenic $\gamma$-ray flux. This model is compatible with the spectrum and composition data at all energies (Globus et al., 2015a,b, hereafter G15a, G15b), and it is consistent with the anisotropy constraints on galactic protons (Tinyakov et al., 2016).

\section{Source model and propagated cosmic-ray spectrum}

Any phenomenological EGCR model that account for the data needs a very hard spectrum at the sources, to reproduce the evolution of the composition above the ankle observed by Auger, and a softer proton component, to account for the light ankle seen by KASCADE-Grande.

The effective spectrum from the mixed composition model (G15b) is displayed in the left panel of Fig. 1. The much softer source spectrum for the nucleons is due to the free escape of neutrons 

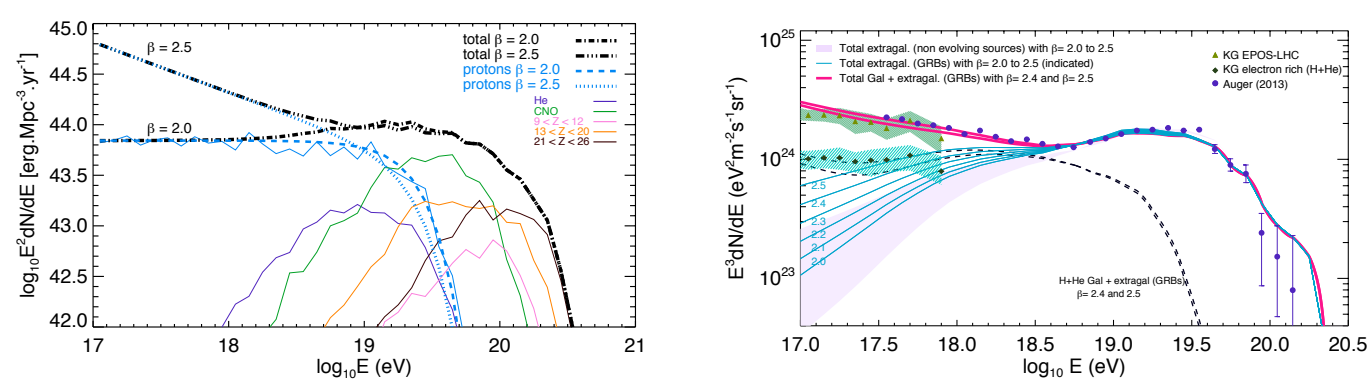

Figure 1: Left panel: UHECR injection spectrum for the various nuclei, as obtained in G15a, with the fit of the proton component with spectral index $\beta=2.0$ (dashed blue), and with its modified shape in the case of $\beta=2.5$. Right panel: Propagated UHECR spectra for $2.0 \leq \beta \leq 2.5$, compared to KASCADE-Grande and Auger data, for a GRB-like cosmological evolution (blue lines) or non-evolving sources (violet shaded area). The total (GCR+EGCR) light component is compared to that deduced from KASCADE-Grande data (using the EPOS-LHC (Wernel et al., 2006) hadronic model), for GRB-like evolution with $\beta=2.4$ and 2.5 (dashed lines).

produced by the photo-disintegration of nuclei at the source, and its exact shape depends on various physical parameters (see G15a for details).

Since the extragalactic protons around $10^{17} \mathrm{eV}$ contribute significantly to the expected cosmogenic $\gamma$-ray flux in the Fermi energy range, we explore, for the sake of generality, (i) different slopes for the proton component $\beta$ (as could result from different physical parameters describing the sources) while keeping the same maximal rigidity and spectral shape for heavier nuclei; (ii) different cosmological evolutions, assuming an average source power proportional to $(1+z)^{\alpha}$ up to a redshift $z_{\max }$. We consider a range of spectral indices $2.0 \leq \beta \leq 2.5$. The two proton spectra with the extreme values of $\beta$ are represented by thick dashed and dotted blue lines, respectively. The implied range of UHECR emissivities above $10^{17} \mathrm{eV}$ is $L_{\mathrm{CR}}^{17} \sim[5.7-14] \cdot 10^{44} \mathrm{erg} \mathrm{Mpc}^{-3} \mathrm{yr}^{-1}$.

The right panel of Fig. 1 depicts the propagated UHECR spectra for $2.0 \leq \beta \leq 2.5$, for EGCR sources evolving as GRBs (Wanderman \& Piran, 2010, blue lines) and for non evolving sources (violet shaded area). In the case of a GRB-like or SFR-like cosmological evolutions, proton spectral indices $\beta \simeq 2.4-2.5$ provide a good fit to the KASCADE-Grande data when summing the light EGCR component with the GCR light component obtained in G15b (dashed line in the right panel of Fig. 1). Softer proton indices are required in the case of a non-evolving scenario.

\section{Gamma-ray and neutrinos counterparts}

The interactions of the propagating EGCRs leads to the production of cosmogenic $\gamma$-rays in the $\mathrm{GeV}-\mathrm{TeV}$ range, and $v \mathrm{~s}$ in the $\mathrm{PeV}-\mathrm{EeV}$ range, through the development of electromagnetic cascades. The Monte-Carlo procedure used to calculate the cosmic-ray, $v$ and $\gamma$-ray spectra is presented in Decerprit \& Allard (2011).

The cosmogenic $\gamma$-rays spectra corresponding to the UHECR spectra of Fig. 1, are shown in Fig. 2 for a mixed-composition model with proton spectral indices $2.0 \leq \beta \leq 2.5$, for sources with no cosmological evolution (violet lines) and with a GRB-like evolution (in blue). These $\gamma$-ray fluxes represent only a small contribution to the total EGB, which is reproduced from Ackermann 

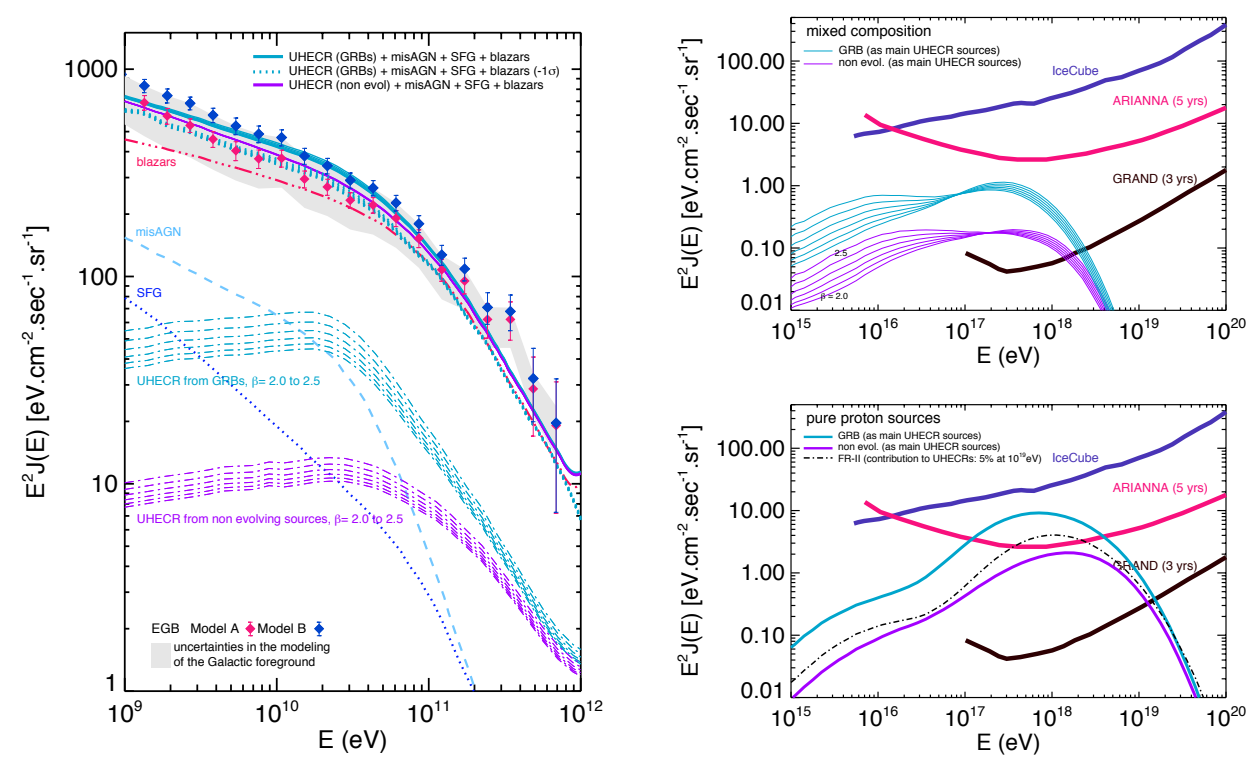

Figure 2: Left panel: $\gamma$-ray fluxes from EGCRs (dashed-dotted lines), for GRB-like evolution (blue) and non-evolving (violet) sources, as computed with our mixed-composition model and spectral indices of the soft proton component $2.0 \leq \beta \leq 2.5$. Also represented the $\gamma$-ray fluxes from SFG, MisAGN and blazar sources (see labels) as modelled by Ackermann et al. (2012); Inoue (2011); Ajello et al. (2015) respectively. The corresponding sum of UHECR, SFG, misAGN and blazar components is represented by thick solid lines (or with a dotted line when 1- $\sigma$ lower bound are adopted for the SFG+misAGN+blazar model, see Ajello et al., 2015), and compared to the EGB estimated from Fermi-LAT data, for both foreground models A and B. Right upper panel: cosmogenic $v$ fluxes associated with our mixed-composition scenarios in the case of GRB-like evolution (blue) and non-evolving (violet) sources, compared with the current IceCube sensitivity (Aartsen et al., 2016) and the expected sensitivities of ARIANNA (5 years, $50 \mathrm{MHz}$ option, Hallgren (2016)) and GRAND (3 years, Martineau-Huynh et al. (2015)). Right lower panel: same, i) for $100 \%$ proton scenarios compatible with the Fermi constraints (plain lines, same colour code), and ii) for a sub-dominant proton component (contributing 5\% of the UHECRs at $10 \mathrm{EeV}$ ) evolving as FR-II galaxies (dashed-dotted line) (Wall et al., 2005).

et al. (2015) for two different models of the Galactic $\gamma$-ray foreground, referred to as model A and model B by the authors. These two models roughly differ by $\sim 20-30 \%$, which can be seen as a rough estimate of their systematics in the subtraction process.

To determine whether a given EGCR source model is compatible with the $\gamma$-ray data, we need to take into account other known contributions to the EGB. The contribution of star-forming galaxies (SFG) and misaligned active galactic nuclei (misAGN), based on the models by Inoue (2011) and Ackermann et al. (2012), are shown in the left panel of Fig. 2 (omitting the uncertainty bands for clarity). Also shown is the $\gamma$-ray spectrum arising from blazars, adapted from Ajello et al. (2015), which appears in good agreement with the PS contribution estimated by A16 and Z16 over the whole energy range. We find that, for the SFR and weaker evolving scenarios, the sum of all components (UHECR, misAGN, SFG and blazars) never exceeds the total EGB, in the case of model B. In the case of model A, the sum is above the EGB. However, it falls below it if one adopts the $1 \sigma$ lower bound on the misAGN+SFG+blazars contribution (see Globus et al., 2017, for the 
estimates of the fluxes of the different contributions in the same energy bands as in A16 and Z16).

The right panel of Fig. 2 shows the resulting $v$ spectra for different EGCR models, together with the sensitivity of current and planned experiments. The mixed-composition models predict $v$ fluxes too low to be detected by IceCube (Aartsen et al., 2016) or ARIANNA (Hallgren, 2016), even in the case of a GRB-like cosmological evolution. They would require a sensitivity such as that expected for the GRAND observatory (Martineau-Huynh et al., 2015) or CHANT satellite concept (Neronov et al., 2016). Pure proton scenarios can be seen on Fig. 2 to yield detectable fluxes by the future ARIANNA experiment, while still being allowed by the current IceCube limits and FermiLAT data. (For these calculations, we assumed a pure proton $E^{-2}$ spectrum with an exponential cutoff at $E_{\max }=60 \mathrm{EeV}$, which is known to reproduce reasonably well the Auger spectrum above the ankle). It is interesting to note that the $v$ s that could be produced by a hypothetical subdominant EGCR proton source, with large enough $E_{\max }$ and cosmological evolution, would contribute a detectable $v$ flux around $10^{18} \mathrm{eV}$, thus making $\mathrm{EeV} v \mathrm{~s}$ a powerful probe for revealing the existence of trans-GZK proton accelerators, even if they do not dominate the observed UHECR flux.

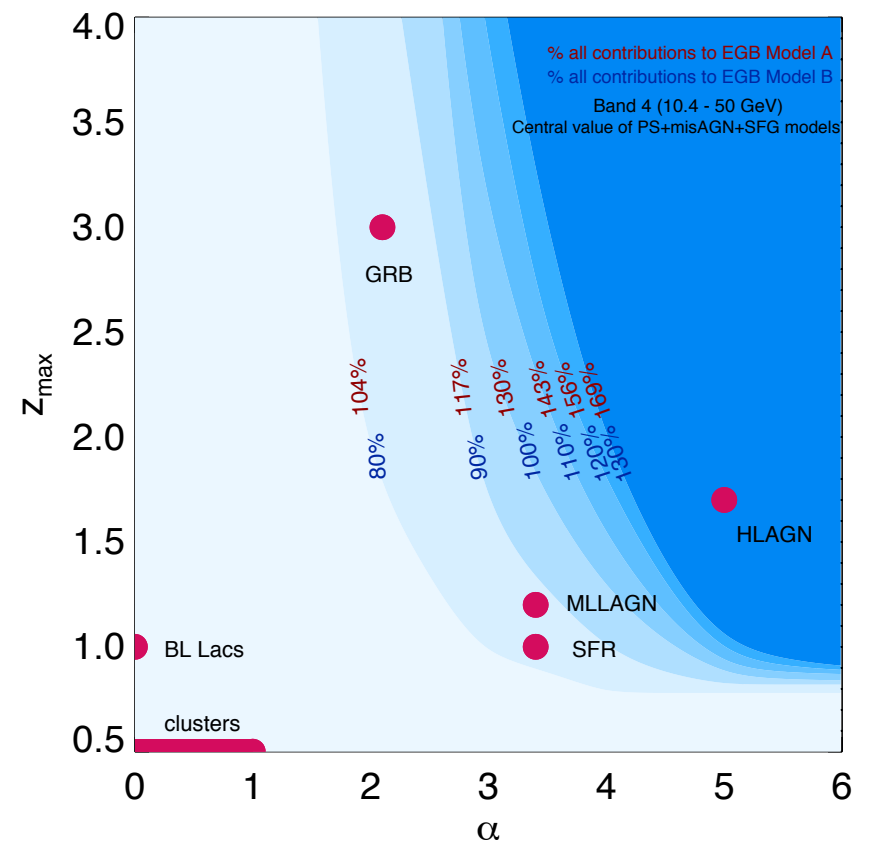

Figure 3: Fermi-LAT constraints on EGCR source evolution in the case of our mixed-composition scenario and proton index $\beta=2.5$. The different colors show the percentage of the sum of all components (UHECR+PS+misAGN+SFG) to the EGB (Models A and B) in the 10.4-50 GeV energy band, in the $\left(\alpha, z_{\max }\right)$ parameter space, where $z_{\max }$ is the maximum redshift up to which sources experience a cosmological evolution in $(1+z)^{\alpha}$. Some possible EGCR sources (see e.g. Gavish \& Eichler, 2016, for the references to the cosmological evolutions) are shown. GRB: gamma-ray bursts. SFR: star-formation rate. MLLAGN: Medium-low-luminosity AGNs. MHLAGN: Medium-High-Luminosity AGNs. HLAGN: High Luminosity AGNs. 


\section{Conclusions}

The UHECR model considered in G15b gives a coherent picture of the GCR-to-EGCR transition, and appears to be compatible with the Fermi-LAT measurements and the estimates of the PS contributions by A16 and Z16. The mixed-composition model appear to be less constrained by the Fermi-LAT than the electron-positron dip (pure-proton) scenario (Berezinsky et al., 2016; Supanitsky, 2016; Gavish \& Eichler, 2016) which is incompatible with the Fermi limits for SFR-like and stronger cosmological evolutions (see also Heinze et al., 2016, for more radical conclusions on the dip model).

Our results are summarized in Fig. 3, that shows the allowed parameter space of different evolutionary scenarios for the mixed composition model. This estimate is based on the summed contribution of all components in the $10.4-50 \mathrm{GeV}$ band, where the contribution from UHECRs is the largest. Only very strong evolutions, e.g. similar to the very luminous AGNs, are excluded by the current observations.

For the evolutionary models allowed by Fermi, the $v$ s fluxes above $10^{17} \mathrm{eV}$ associated with the mixed-composition scenario are well below the current IceCube limits. These fluxes are within the reach only of the most sensitive planed $v$ observatories.

Finally, we note that while the PS contributions are now understood to dominate the extragalactic $\gamma$-ray fluxes in the $\mathrm{GeV}-\mathrm{TeV}$ range, the uncertainties on the different contributions (notably for sources other than blazars, see e.g Di Mauro et al., 2013; Lacki et al., 2014; Tamborra et al., 2014) as well as on the Galactic foreground are still too large to efficiently constrain the cosmological evolution of UHECR sources. Since the $\gamma$-ray fluxes associated with mixed-composition UHECRs never exceed $\sim 20 \%$ of the EGB (at least for source evolutions not significantly larger than SFR, see Table 2 in Globus et al. (2017)), the EGB and its other contributions should be determined to this level of precision in order to estimate whether a UHECR mixed-composition model is excluded. Moreover, the Fermi-LAT estimates of the Galactic foreground are based on the GALPROP framework (Strong et al., 2000). These calculations rely on several simplifying assumptions in particular in the description of the Galactic cosmic-ray source distribution or the magnetic halo, as well as on several ad-hoc parameters that are tuned to reproduce cosmic-ray data. Alternative models (e.g. Nava et al., 2017, and references therein) have been shown to fairly account for the primary-tosecondary ratios as well as some puzzling features in the observed $\gamma$-ray Galactic signal. These models have a smaller halo extension and would probably result in a lower Galactic foreground, leaving more room for EGCR contributions.

\section{Acknowledgments}

NG and TP acknowledges the I-CORE Program of the Planning and Budgeting Committee and The Israel Science Foundation (grant 1829/12), the advanced ERC grant TReX, and the Lady Davis foundation.

\section{References}

Aab A. et al. (Pierre Auger Collaboration), 2014, Phys. Rev. D 90, 122006 
Aartsen, M. G. et al. [IceCube collaboration], 2016, Phys. Rev. Lett. 117 (24), 241101, arXiv:1607.05886 [astro-ph.HE]

Ackermann M. e. a., 2015, ApJ, 799, 86

Ackermann M. e. a., 2016, Phys Rev Letters,116, 151105

Ackermann, M., Ajello, M., Allafort, A., et al. 2012b, ApJ, 755, 164

Ahlers M., Anchordoqui L. A. and Sarkar S., 2009, Phys. Rev. D 79(8), 083009.

Ahlers M., Salvado J., 2011, Phys.Rev. D84, 085019

Ajello, M., Gasparrini, D., Sánchez-Conde, M. , Zaharijas, G., Gustafsson M., et al., 2015, ApJ Letters, 800:L27

Allard D., Ave M., Busca N., Malkan M. A., Olinto A. V., Parizot E., Stecker F. W. and Yamamoto T., 2006, Journal of Cosmology and Astro-Particle Physics 9, 5.

Anchordoqui L. A., Goldberg H., Hooper D., Sarkar S., Taylor A., 2007, Phys. Rev. D, vol. 76 pp. 123008

W. Apel et al. (KASCADE-Grande Collaboration), Nucl. Instrum. Methods Phys. Res., Sect. A 620, 202 (2010).

W. Apel et al. (KASCADE-Grande Collaboration),Phys. Rev. D 87081101 (2013).

Berezinsky V. S., Gazizov A. \& Kalashev O., 2016, Astropart. Phys. 84, 52

M. Bertaina et al., 2015, proc. of the 34th Int. Cosmic Ray Conf., The Hague, The Netherlands

Coppi, P. S., Aharonian, F. A., 1997, ApJ, 487, 9

Decerprit G., Allard D., 2011, A\&A, 535, A66

Di Mauro, M., Calore, F., Donato, F., Ajello, M., \& Latronico, L. 2013, ApJ, 780, 161

Engel R., Seckel D. and Stanev T., 2001, Phys. Rev. D 64(9), 093010.

Gavish E., Eichler D., 2016, ApJ, 822, 56

Globus N., Allard D., Mochkovitch R., Parizot E., 2015, MNRAS, 451, 751

Globus N., Allard D., Parizot E., 2015a, Phys Rev D, 92, 021302

Globus N., Allard D., Parizot E., Piran, T., 2017, ApJ, 839, 22

Hallgren A. for the ARIANNA collaboration, 2016, EPJ Web of Conferences, 11603003

Heinze J., Boncioli D., Bustamante M., Winter W., 2016, ApJ, 825, 122

Inoue, Y. 2011, ApJ, 733, 66 
Kotera K., Allard D. and Olinto A. V., 2010, Journal of Cosmology and Astroparticle Physics, vol. 10 pp. 013

Lacki, B. C., Horiuchi, S., \& Beacom, J. F. 2014, ApJ, 786, 40

Malyshev D. \& Hogg D. W., 2011, ApJ 738, 181

Martineau-Huynh O. et al., 2015, Proceedings of The 34th International Cosmic Ray Conference, The Hague, The Netherlands, arXiv:1508.01919

Nava, L., Benyamin, D., Piran, T. \& Shaviv, N. J., 2017, MNRAS, 466, 3674

Neronov A. et al., 2016, arXiv:1606.03629

Protheroe R. J and Johnson P. A., 1996, Astroparticle Physics, vol. 4 pp. 253

Seckel D. and Stanev T., 2005, Physical Review Letters 95(14), 141101

Strong, A. W., Moskalenko, I. V., \& Reimer, O., 2000, ApJ, 537, 763

Stecker F. W., 1979 ApJ 228, 919

Supanitsky A. D., 2016, Phys. Rev. D 94, 063002

Tamborra, I., Ando, S., \& Murase, K. 2014, J. Cosmology Astropart. Phys., 9, 43

Tinyakov, P. G., Urban, F. R., Ivanov, D., Thomson, G. B., Tirone, A. H., 2016, MNRAS, 460, 3479

Wall J. V., Jackson C. A., Shaver P. A., Hook I. M. and Kellermann K. I., 2005, A\&A 434, 133

Wanderman D., Piran T., 2010, MNRAS, 406, 1944

K. Werner, M. F. Liu \&T. Pierog, 2006, Phys. Rev. C74 044902

Zechlin, H-S et al., 2016, ApJ Letters, 826, L31 